\title{
CHP-Brentuximab Vedotin Regimen
}

National Cancer Institute

\section{Source}

National Cancer Institute. CHP-Brentuximab Vedotin Regimen. NCI Thesaurus. Code C159558.

A regimen consisting of brentuximab vedotin, cyclophosphamide, doxorubicin and prednisone that can be used to treat CD30-expressing peripheral T-cell lymphomas. 